\title{
MAMÍFEROS DE LA RESERVA DE LA BIOSFERA LA SEPULTURA, CHIAPAS, MÉXICO
}

\author{
EDUARDO ESPINOZA MEDINILLA, EPIGMENIO CRUZ², IVÁN LIRA ${ }^{3}$, IG- \\ NACIO SÁNCHEZ ${ }^{1}$.
}

\author{
1 El Colegio de la Frontera Sur. Carr. Panamericana y Periférico Sur Barrio de Ma. Auxili- \\ adora, San Cristóbal de las Casas, Chiapas. \\ e-Mail:emedinilla@sclc.ecosur.mx \\ 2 Instituto de Historia Natural y Ecología. ZooMAT. Calz. Cerro Hueco S/N Col. El Zapotal. \\ Apdo. Post. 6 Tuxtla Gutiérrez, Chiapas, México C.P.29000. \\ e-Mail:cruz@chiapas.net
}

3 Palma Viajero 411, Col. Las Palmas, C.P. 29040, Tuxtla Gutiérrez, Chiapas, México. e-Mail: ilira@terra.com.mx

\begin{abstract}
Resumen: Con el objeto de conocer la riqueza de mamíferos de la Reserva de la Biosfera La Sepultura, fue elaborado un listado de especies para esta área natural protegida, mediante transectos lineales realizándose observaciones directas, búsqueda de rastros y captura de ejemplares, durante el periodo de mayo de 1994 a diciembre de 1999. Se realizó una revisión de bases de datos nacionales y extranjeras con registros de esta localidad. Se recolectaron 848 especímenes (248 pieles y cráneos), 32 huellas en molde de yeso y nueve fragmentos óseos. Se obtuvo un listado compuesto de 98 especies, 70 géneros, 29 familias y 10 órdenes. El $86.7 \%$ de estas especies pertenecen a los órdenes Chiroptera, Rodentia y Carnivora. 21 especies están clasificadas dentro de alguna categoría de conservación. Los datos obtenidos de este manuscrito constituyen una base de datos sólida y actualizada que pueden ser usadas como parte de los planes de manejo y conservación para esta reserva.
\end{abstract}

\begin{abstract}
In order to determine the mammalian richness of La Sepultura Biosphere Reserve, a species list was prepared for this Natural Protected Area. Line transects were conducted and data gathered troughout, along line transects, recording direct observations, animal sings, and capture, during beween May 1994 - December 1999. Also, national and international databases where revised. A total of 848 specimens (248 furs and skulls), 32 plaster tracks, and 9 bone fragments were collected. A list of 98 species, 70 genera, 29 families, and 10 orders was elaborated. Of these species, $87.7 \%$ belong to the orders Chiroptera, Rodentia and Carnivora. 21 species have some type of legal protection. The results obtained in this manuscript form a solid and updated database, which can be used in the of management and conservation plans for this Reserve.
\end{abstract}

Palabras Claves: Chiapas, Mamíferos, México, Reserva de la Biosfera La Sepultura. 


\section{INTRODUCCIÓN}

La Reserva de la Biosfera de La Sepultura, situada al noroeste de Chiapas, México, abarca una superficie de 192, 734 ha. La heterogeneidad altitudinal, topográfica, climática y edáfica de la región ha redituado en una gran variedad de ecosistemas, encontrándose 8 de los 18 tipos de vegetación primaria del estado de Chiapas, México: bosque lluvioso de montaña, bosque de niebla, chaparral de niebla, bosque de pinoencino, bosque de pino, selva mediana subperennifolia y subcaducifolia, selva baja caducifolia, así como la presencia de potreros, milpas de maíz y fríjol, y cafeticultura orgánica (Breedlove, 1981). En los estudios iniciales, para su establecimiento como reserva, se habían registrado 49 especies de mamíferos, 143 especies de aves, 48 especies de reptiles así como numerosas especies de anfibios, peces e invertebrados no determinados (Hernández, 1994).

Los listados de flora y fauna proveen información sólida y confiable sobre cambios en las poblaciones, su fenología y patrones de abundancia geográfica a escala local, regional y global (Droege et al., 1998). Por lo anterior se consideró que con base en la información de colecciones nacionales y extranjeras, publicaciones y trabajos de campo, se elaborara un listado que permitiera proponer medidas y criterios de conservación congruentes hacia la fauna de mamíferos que esta reserva contiene.

\section{MÉTODOS}

La Reserva de la Biosfera La Sepultura (REBISE), es decretada el 5 de junio de 1995, con una extensión de 192, 734 ha, se ubica en el Estado de Chiapas entre los $16^{\circ} 00^{\prime}$ y $16^{\circ} 29^{\prime}$ latitud Norte y $93^{\circ} 24^{\prime}$ y $94^{\circ} 07^{\prime}$ longitud Oeste, dentro de la región fisiográfica de la Sierra Madre de Chiapas, región sumamente accidentada que se eleva desde los 25 hasta los 2, $550 \mathrm{msnm}$ altura máxima alcanzada por el Cerro Tres Picos (INE-CONABIO, 1995). Para esta región se distinguen dos zonas: una húmeda, caracterizada por una alta precipitación pluvial, que abarca la porción Este de la comarca El Soconusco, con selvas medianas y bosque mesófilo de montaña. Y ésta se caracteriza por ser menos húmeda, e incluye una porción del Istmo de Tehuantepec, que según Goodwin (1969) es una de las regiones más secas del Pacífico, por lo que las asociaciones vegetales características son las selvas bajas caducifolias y matorrales xerófilos (Breedlove 1981, Miranda, 1975). En su conjunto, la reserva es considerada como una zona de alta diversidad biológica y elevado endemismo (Hernández, 1994).

Durante los últimos doce años, personal del Instituto de Historia Natural y Ecología de Chiapas (I.H.N.E.) ha realizado visitas periódicas con el fin de obtener un listado completo sobre la fauna del lugar (IHN, 1991). Pero fue a partir de mayo en 1994 
que se comenzó con una serie de visitas sistemáticas que incluyen el establecimiento de 20 transectos lineales, con longitudes que varían entre los 0.7 a $12 \mathrm{~km}$ de longitud y, 1 a $5 \mathrm{~m}$ de ancho, en siete tipos de hábitat.

Los transectos se recorrieron cuando menos una vez por mes, registrando todas las huellas, excretas, restos óseos, echaderos, así como observaciones directas, tanto diurnas como nocturnas y, procurando evitar sitios con suelo demasiado compacto o pedregoso que impidiera la impresión o recolecta de rastros como señala Aranda (1980).

Los mamíferos de talla pequeña (Musarañas, Murciélagos y Ratones), fueron muestreados con 100 trampas Sherman y 20 Tomahawk, colocadas en los transectos. Entre las 18:00 y las 01:00 horas se instalaron redes de niebla de $11 \mathrm{~m}$ de longitud, en número variable de acuerdo a la localidad, no siendo menor de tres y un máximo de seis redes por noche.

Los ejemplares fueron preparados de acuerdo a la técnica de Ramírez Pulido et al. (1989). La determinación taxonómica se llevó a cabo de acuerdo a Hall (1981), Medellín et al. (1997) y Villa (1966). En algunas determinaciones fue necesaria la comparación con ejemplares de la Colección Nacional de Mamíferos del Instituto de Biología de la Universidad Nacional Autónoma de México. Todos los ejemplares fueron depositados en la Colección Zoológica Regional del Sureste de México Miguel Álvarez del Toro del Instituto de Historia Natural y Ecología del Estado de Chiapas.

Fueron consultadas las bases de datos en 26 museos nacionales y extranjeros, referente a todos los especimenes que se encuentran en su acervo para el estado de Chiapas, de éstos, se registraron las localidades específicas dentro del área de la reserva. Una vez verificada esta información, se integraron las especies a nuestro listado. Con base a éste se realizaron tres tipos de análisis; el primero sobre la afinidad biogeográfica de cada una de las especies, el segundo por su tipo de alimentación y el tercero por el estado de conservación de acuerdo a Arita (1993), Arita y Ceballos (1997), NOM059-ECOL-2001 Secretaria de Medio Ambiente y Recursos Naturales (SEMARNAT, 2001) y de Unión Internacional para la Conservación de la Naturaleza y los Recursos Naturales (UICN, 2002).

\section{RESULTADOS}

Se capturaron 848 ejemplares de mamíferos, de los cuales 590 (69.5\%) fueron liberados y 258 preparados como ejemplares de museo. También se registraron 32 huellas en moldes de yeso y nueve restos óseos. Durante los recorridos tanto diurnos como nocturnos se realizaron observaciones directas de algunas especies. De los museos consultados, 11 contenían ejemplares colectados en esta zona, por lo que se obtuvieron 456 registros para la reserva, de los cuales la mayor parte corresponden al periodo de 1950 a 1970.

Se obtuvieron 98 especies de mamíferos (Anexo 1), incluyendo el primer registro para el Estado de Chiapas de Marmosa canescens (ratón tlacuache). Las es- 
pecies se encuentran agrupadas en 10 órdenes, 29 familias y 70 géneros. Los órdenes con mayor representación de especies fueron el Chiroptera, Rodentia y Carnivora con un $86.7 \%$ del total de las especies. Considerando que México tiene 452 especies de mamíferos terrestres (Arita y Ceballos, 1997) la REBISE incluye el

$21.6 \%$ del total de especies, mientras que el de Chiapas, incluye el $49.4 \%$ del total de las especies de México (Fig. 1).

El orden con mayor número de especies es el Chiroptera, de los cuales el $91.02 \%$ pertenecen a las familias Phyllostomidae (60\%), Vespertilionidae $(17.7 \%)$, Emballonuridae (6.6\%) y Mormoopidae (6.6\%).

Para el orden Rodentia, el segundo en mayor representatividad, el $73.8 \%$ pertenecen a las familias Sciuridae (13.04\%), Heteromyidae (13.04\%) y Muridae (47.8 \%). En el caso de los carnívoros las familias con mayor representatividad fueron la Procyonidae (23.5 \%), Mustelidae (35.2\%), y Felidae (29.4 \%).

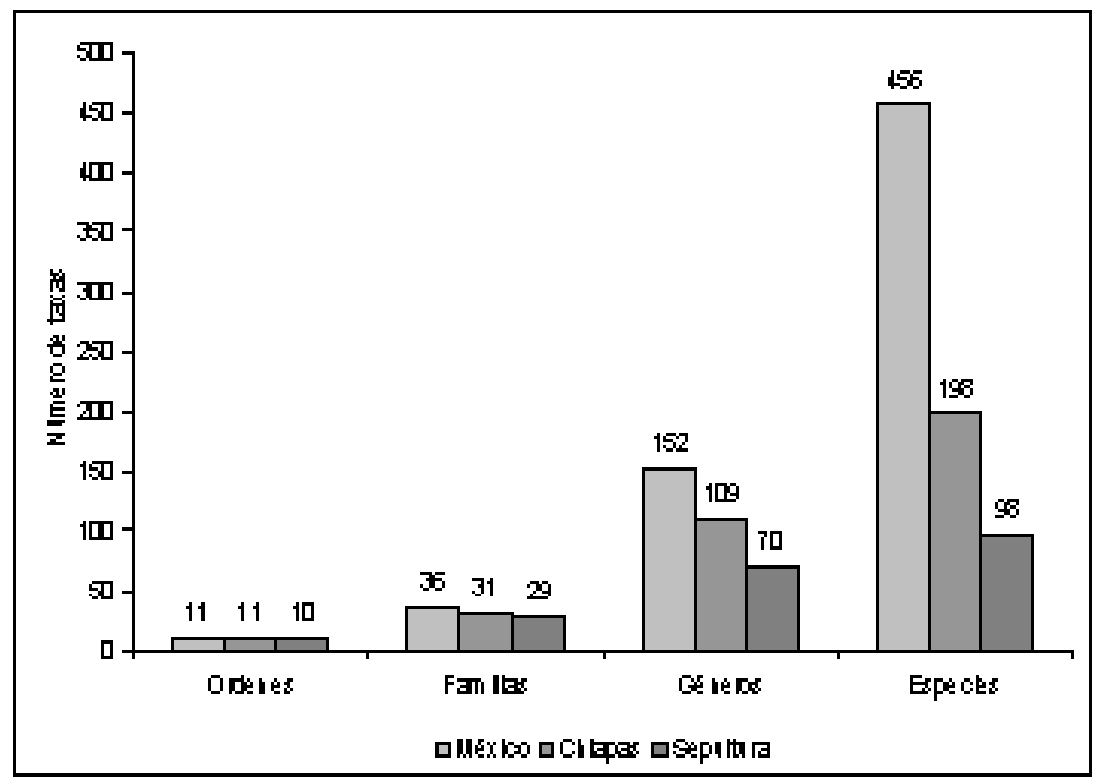

Figura 1. Comparación de la riqueza mastofaunística de México, Estado de Chiapas y la REBISE. 
Con respecto a su origen, la mastofauna de la reserva es principalmente Neotropical (NT) con un $50 \%$. Las especies compartidas entre Norte y Sudamérica (NS) son el $29 \%$, las especies Mesoamericanas (MA) $13 \%$ y con un bajo nivel de filiación entre las endémicas a México (MX) con $4 \%$ y las Neárticas en un $4 \%$ (EA) (Fig. 2).

De acuerdo a su forma de alimentación, los mamíferos de la REBISE se pueden agrupar en 14 diferentes categoría. Un porcentaje elevado de especies, el 44.32 $\%$, son frugívoro-omnívoro (FO, 43 especies), seguidos de los insectívoro-omnívoro representados por un $21.64 \%$ (IO, 21 especies), los frugívoro-granívoros con un 12.37 $\%$ (FG, 12 especies), los carnívoros con un $6.18 \%$ (CA, 6 especies), los hematófagos con un $2.06 \%$ (H, 2 especies), los frugívoro-herbívoros con un $4.12 \%$ (FH, 4 especies), los carnívoro-omnívoros con un $2.06 \%$ (CA/O, 2 especies), y los piscívoros $(\mathrm{P})$, carnívoro-piscívoros (CA/P), los nectarívoros (NE), los myrmecophagos (MY), herbívoro-pasteador (HZ), los herbívoro-ramoneadores (HB), los raizívoros (RH), y los insectívoros (I), estas siete categorías de alimentación representan respectivamente el $1.03 \%$ (Fig. 3).

Por lo menos 20 especies se encuentran clasificadas en alguna categoría de riesgo de extinción (Anexo 1). Al menos nueve especies de mamíferos de La Sepultura se encuentran en la lista roja de la Unión Internacional para la Conservación de la Naturaleza (IUCN/2002). La Convención Internacional sobre Tráfico de Especies en Peligro de Flora y Fauna (CITES) considera a 15 especies dentro de alguno de sus tres apéndices, muy similar a lo propuesto por la Norma Oficial Mexicana (SEMARNAT NOM-059-ECOL-2001), donde sólo 15 especies presentan algún criterio de conservación. Parece no existir concordancia entre los criterios empleados por cada una de estas instancias, pues solamente ocho especies son consideradas por al menos dos de ellas (Fig. 4).

Al menos 23 especies de mamíferos son aprovechadas de alguna manera por los pobladores de la región; 14 especies son utilizadas casi exclusivamente para consumo local, ya sea como alimento o como mascotas. Entre las más comunes se encuentran Didelphis marsupialis, D. virginiana, Tapirus bairdii, Agouti paca, Dasyprocta mexicana, Sylvilagus floridanus, Ateles geoffroyi, Odocoileus virginianus, Mazama americana, Peccari tajacu y Nasua narica. Con fines ornamentales o como mascotas se utilizan: Herpailurus yagouaroundi, Leopardus pardalis, Leopardus wiedii, Ateles geoffroyi, y Procyon lotor o con ambos propósitos: Potos flavus y Sciurus aureogaster, Nasua narica, Procyon lotor (Cruz, 2000).

Dasypus novemcinctus y Ateles geoffroyi son las especies a las que se les da un número mayor de usos, pues además de ser utilizados como alimento, adornos o mascotas por la gente local, son comercializados por creer que tienen propiedades medicinales (Cruz, 2000). 


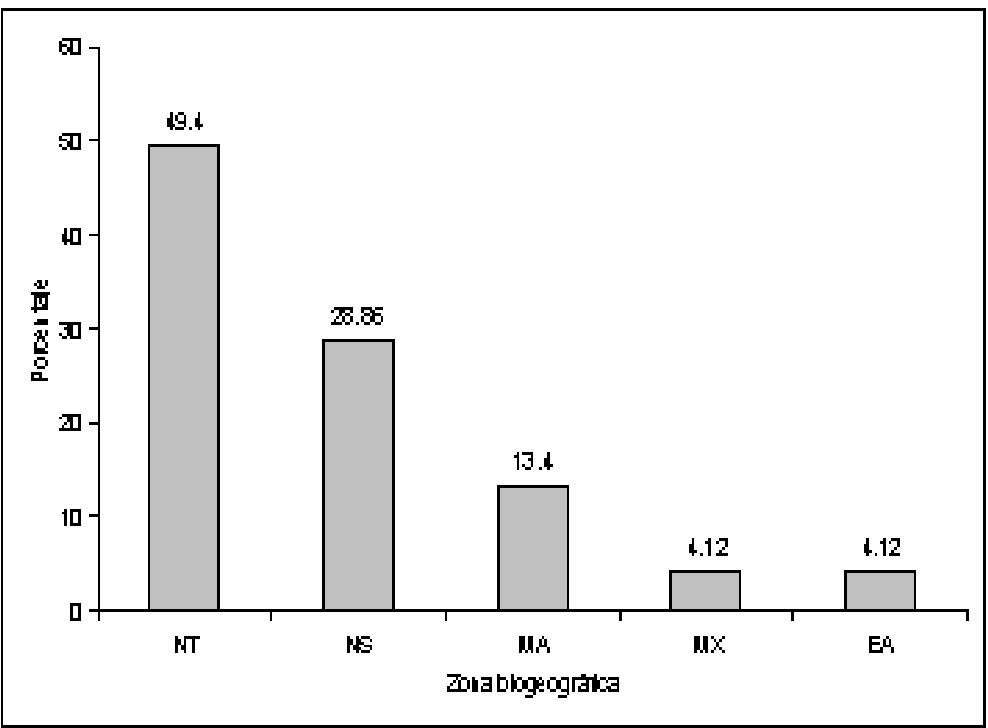

Figura 2. Origen de las especies de acuerdo a su afinidad biogeográfica. NT= Neotropical; $\mathrm{NS}=$ Compartidas entre Norte y Sudamérica; $\mathrm{MA}=$ Mesoamericanas; $\mathrm{MX}=$ Endémicas de México; EA= Neárticas.

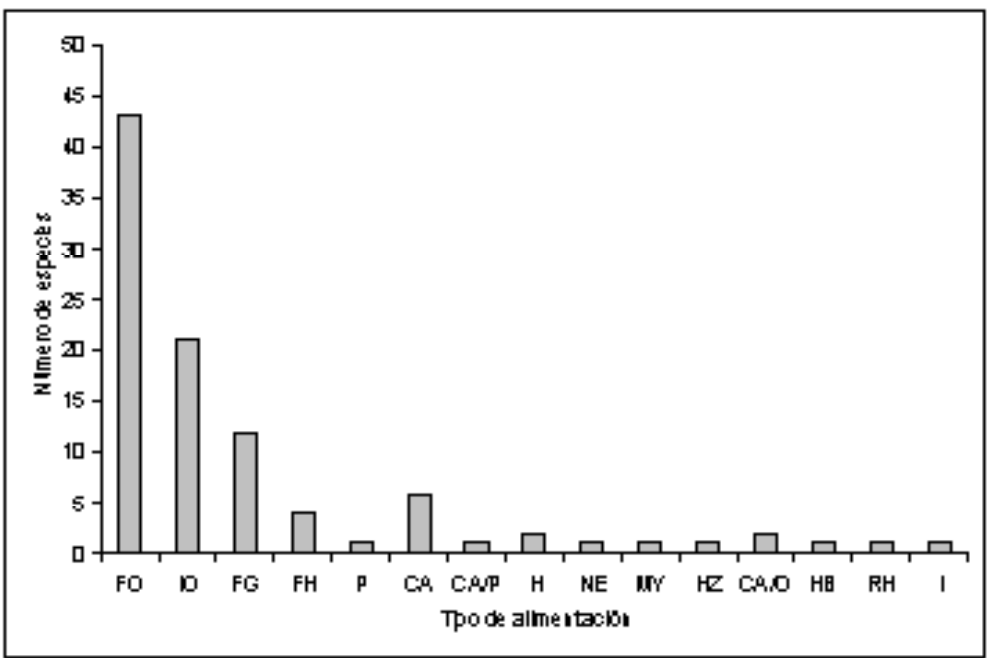

Figura 3. Tipos de alimentación de los mamíferos en la REBISE. 


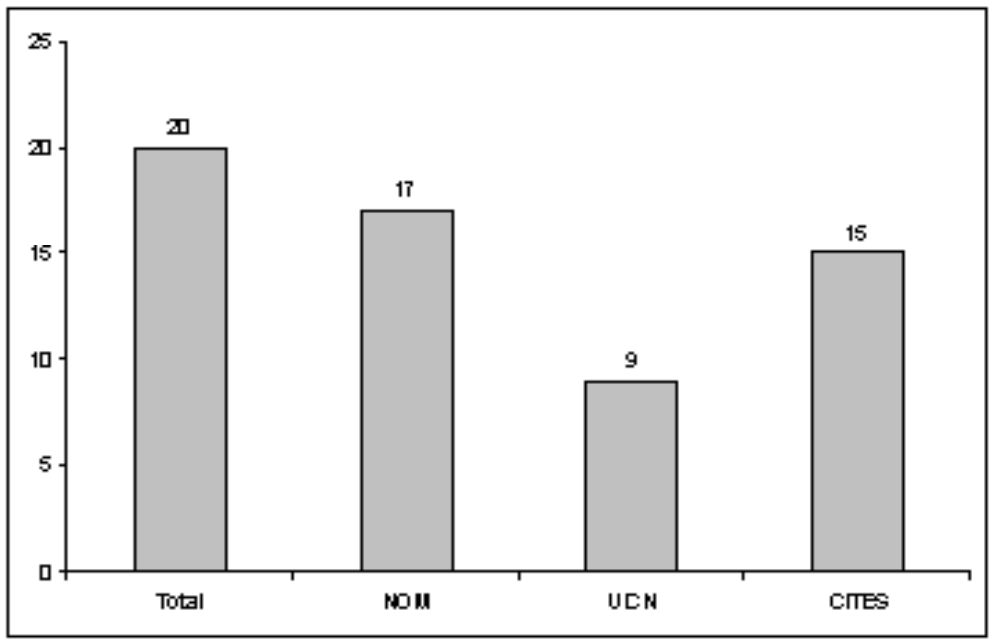

Figura 4. Comparación de la riqueza mastofaunística de la REBISE con otras áreas naturales protegidas en el Estado de Chiapas.

\section{DISCUSIÓN}

La mastofauna de la Reserva de la Biosfera La Sepultura se encuentra representada por un total de 98 especies, con este estudio se han aportado 37 nuevos registros a los 61 que se tenían para el área. De particular importancia es el registro de Marmosa canescens ya que aumenta el rango de distribución de la especies hasta el Estado de Chiapas, probablemente siguiendo la distribución de la selva baja caducifolia.

De acuerdo a listados mastofaunísticos que se tienen de otras áreas naturales protegidas en el estado, se puede observar que la REBISE ocupa el tercer lugar en cuanto a número de especies registradas, seguida por la Selva El Ocote con 97 especies (Navarrete et al., 1996), el Cañón del Sumidero con 46 especies (Gálvez, 1990), siendo El Triunfo con 112 especies (Espinoza et al., 1998) y la Selva Lacandona con 124 especies (March y Aranda, 1992) las reservas que albergan la mayor diversidad de especies (Fig. 5).

Entre los principales problemas de la región se encuentran: la expansión de la frontera agropecuaria, los incendios forestales y la creación de nuevos centros de población. Dentro del área además de las actividades antes mencionadas se realiza un aprovechamiento no regulado de recursos maderables (pino Pinus sp, encino Quercus sp., cedro Cedrella sp., amate Ficus sp.) y no maderables (Palma camedora o Chamaedorea sp.) (INE-SEMARNAP, 1999a).

Frecuentemente se practica la cacería de autoconsumo, la comercial y el tráfico 
de animales. La primera constituye una alternativa de complemento a la dieta familiar y las dos últimas se llevan a cabo como alternativas del ingreso económico. La cacería de autoconsumo se realiza prácticamente por todas las comunidades asentadas dentro de la Sierra; es una práctica comúnmente asociada a la recolección de la palma Chamaedora sp., aunque oficialmente la región presenta veda permanente al aprovechamiento de todas las especies de flora y fauna (INE-SEMARNAP, 1999b).

\section{CONCLUSIONES}

Debido a la influencia de las especies compartidas entre Norte y Sudamérica, que aunadas a las especies neárticas la fauna de mamíferos de la REBISE ofrece un panorama único en su conjunto de especies. La riqueza de especies es superior a la presentada por la Reserva de la Biosfera Selva El Ocote y al Parque Nacional Cañón del Sumidero.

En vista de que una alta proporción de especies enfrenta algún tipo de amenaza

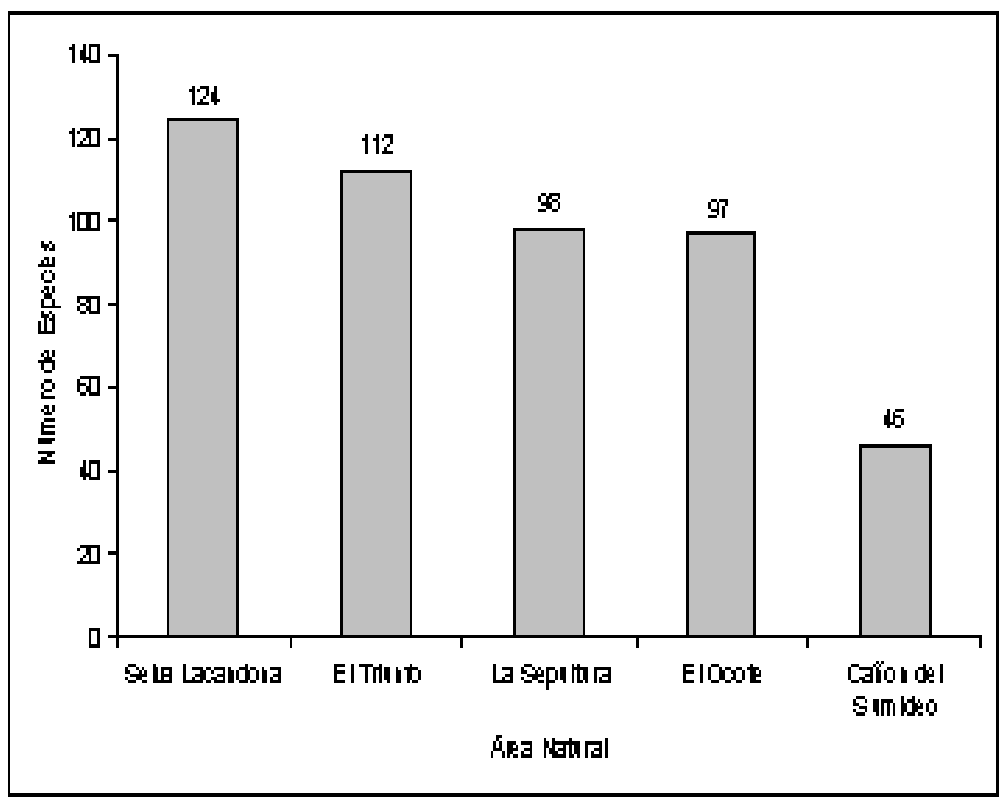

Figura 5. Comparación de la riqueza mastofaunística de la REBISE con otras áreas naturales protegidas en el Estado de Chiapas. 
de extinción, una estrategia de conservación sería el establecimiento de Unidades de Manejo y Aprovechamiento (UMAS), además de la implementación de sistemas agrosilvopastoriles y agroforestales, que en este caso pueden ser más benéficos para la conservación de la fauna silvestre, por lo que es importante conducir investigaciones dirigidas a la integración de estas acciones dentro de la reserva.

Cabe destacar que la lista de mamíferos que se presenta es parcial, falta seguir realizando más estudios y observaciones en el lugar. Principalmente en los roedores, quirópteros e insectívoros. Es necesario resaltar la importancia de la reserva como refugio de comunidades de mamíferos, principalmente de aquellas de distribución restringida y que al parecer, no habitan en otras áreas naturales protegidas de México y Chiapas.

\section{AGRADECIMIENTOS}

Al personal del I.H.N.E. que colaboró en la realización y observaciones en la reserva, principalmente a Antonio Ramírez Velásquez, Fredi M. Herrera Pérez y de manera muy especial a la familia Canseco Cruz, por todas las facilidades y apoyo en los viajes de campo. A todos los revisores anónimos que participaron en la revisión del presente manuscrito, por sus comentarios y observaciones. La mayor parte del trabajo fue financiada por la Comisión Nacional para el Conocimiento y Uso de la Biodiversidad (CONABIO) a través del proyecto P060 y el Proyecto Ecología, Biología y Conservación del Tapir (Tapirus bairdii) en el Estado de Chiapas, perteneciente a la línea de investigación del Instituto de Historia Natural y Ecología.

\section{LITERATURA CITADA}

Álvarez del Toro, M. 1991. Los Mamiferos de Chiapas. Gobierno del Estado de Chiapas. DIF-Chiapas / Instituto Chiapaneco de Cultura. Tuxtla Gutiérrez, Chiapas, México.

Aranda, J.M. 1980. Importancia y utilidad de los rastros para el estudio de mamiferos silvestres. Tesis de licenciatura. Facultad de Ciencias. UNAM. México, D.F. México.

Arita, H. T.1993. Riqueza de Especies de la Mastofauna de México. en: Avances en el Estudio de los Mamiferos de México (R.A. Medellín R. A. y G. Ceballos, eds). Publicaciones Especiales, Asociación Mexicana de Mastozoología, A.C 1;1-464 p.

Arita, H.T. y G. Ceballos. 1997. Los mamíferos de México: Distribución y estado de conservación. Revista Mexicana de Mastozoología, 2:33-71.

Breedlove, D.E. 1981. Flora of Chiapas. Part I. Introduction to the flora of Chiapas. California Academy of Sciences. San Francisco, Cal. USA. California, EUA.

Cruz, A. E. 2000. Usos tradicionales de la fauna silvestre de Chiapas, BARUM, Revista del Instituto de Historia Natural, Chiapas, México. Vol .25. Pp. 25-28.

Espinoza, M., E. A. Anzures, y E. Cruz. 1998. Los Mamíferos de El Triunfo. Revista Mexicana de Mastozoología, 3:79-94. 
Droege, S., A. Cyr, y J. Larivee. 1998. Chechlists: An Under - Used Tood for the Inventory and Monitoring of Plants and Animals. Conservation Biology, 12 (5):1134 - 1138.

Gálvez, J. 1990. Mastofauna del Parque Nacional "Cañón del Sumidero" Chiapas, México. Instituto de Ciencias y Artes de Chipas. Tesis de Licenciatura. Tuxtla Gutiérrez, Chipas.

Goodwin, G.G. 1969. Mammals from the state of Oaxaca, Mexico in America Museum of History. Bulletin of the America Museum of Natural History, 14(1).

Hall, E. R. 1981. The Mammals of North America. Vols. I, II. John Wiley \& Sons. New York, EUA.

Hernández, Y. A. 1994. Propuesta para establecer el área Natural Protegida Reserva de la Biosfera "La Sepultura", en la porción oeste de la Sierra Madre de Chiapas. Tesis de Lic. Universidad Veracruzana Escuela de Biología, Xalapa Veracruz, México.

Instituto de Historia Natural. 1991. Propuesta área natural protegida Zona sujeta a Conservación ecológica "La Sepultura". Resumen ejecutivo. Instituto de Historia Natural, Depto. De Áreas Naturales, Tuxtla Gutiérrez, Chiapas, México.

Instituto Nacional de Ecología. Comisión Nacional para el Conocimiento y Uso de la Biodiversidad. 1995. Reservas de la Biosfera y otras Áreas Naturales Protegidas de México. Secretaria del Medio Ambiente, Recursos Naturales y Pesca. INE - CONABIO, México D.F.

Instituto Nacional de Ecología y Secretaría del Medio Ambiente Recursos Naturales y Pesca. 1999a. Programa de Manejo de la Reserva de La Biosfera El Triunfo, México. INE - SEMARNAP. México D.F.

Instituto Nacional de Ecología y Secretaría del Medio Ambiente Recursos Naturales y Pesca. 1999b. Programa de Manejo de la Reserva de La Biosfera La Sepultura, México. INE SEMARNAP. México D.F.

March, I. y M. Aranda. 1992. Mamíferos de la Selva Lacandona, Pp. 201-220, en: Reserva de la Biosfera Montes Azules, Selva Lacandona: Investigación para su Conservación (Vázquez, M.A.y M.A. Ramos, eds.). Publicaciones Especiales ECOSFERA 1:1-436.

Medellín, R. A., H. T. Arita y O. Sánchez T. 1997. Identificación de los murciélagos de México: Clave de campo. Publicaciones Especiales, Asociación Mexicana de Mastozoología, A.C. México, D. F.

Miranda, F. 1975. La Vegetación de Chiapas. $2^{\mathrm{a}}$ Edición. Ediciones del Gobierno del Estado. Tuxtla Gutiérrez, Chiapas, México.

Navarrete, D. A., M. P. Alba, I. J. March y E. Espinoza. 1996. Mamíferos de la Selva El Ocote, Chiapas. Pp. 179-207. en: Conservación y Desarrollo Sustentable en la Selva El Ocote, Chiapas (M. A. Vázquez. y I. March M. eds.) El Colegio de la Frontera Sur en colaboración del Centro de Estudios para la Conservación de los Recursos Naturales, A.C.

Ramírez-P.J., Lira. I. Salvador Gaona., C. Müdespacher y A. Castro-Campillo. 1989. Manejo y Mantenimiento de Colecciones Mastozoológicas. Universidad Autónoma Metropolitana, México, D.F.

Secretaría de Medio Ambiente Recursos Naturales y Pesca (SEMARNAT). 2001. Norma Oficial Mexicana NOM-059-ECOL-2001. Protección ambiental- especies nativas de México de flora y fauna silvestres- categorías de riesgo y especificaciones para su inclusión, exclusión o cambio- lista de especies en riesgo. Diario Oficial de la Federación 1:1-22.

Unión Internacional para la Conservación de la Naturaleza y los Recursos Naturales (UICN). 
2002. 2000 IUCN Red list of threatened animals. IUCN Species Survival Commission, Gland, Suiza. (http://www.redlist.org).

Villa, R. B. 1966. Los Murciélagos de México. Instituto de Biología, UNAM., México, D.F.

Wilson, D. E. y D. M. Reeder (eds). 1993. Mammal species of the world: A Taxonomic and Geographic Reference. $2^{\text {nd }}$. Ed., Smithsonian Institution Press. Washington, D.C. EUA. 
APÉNDICE 1. Lista preliminar de las especies de mamíferos de la Reserva de la Biosfera "La Sepultura". Los órdenes se mencionan en la secuencia filogenética propuesta por Wilson y Reeder (1993). Las familias, géneros y especies se listan en orden alfabético.

Las abreviaturas en las columnas son como sigue: el nombre común de las especies está basado en las publicaciones realizadas por Alvarez del Toro (1991), DIST: distribución (NT = neotropical; MA = mesoamericano; $\mathrm{MX}$ = endémico de México; $\mathrm{NS}$ = compartidos en Norteamérica y Sudamérica; EA = neártico). Nom: Conservación según NOM-059-ECOL-2000 (E = extinta en medio silvestre; $\mathrm{P}$ = peligro de extinción; $\mathrm{A}=$ amenazada; $\mathrm{PR}=$ sujeta a protección especial). IUCN: Categoría según IUCN (EX = extinta; $\mathrm{EW}=$ extinta en estado silvestre; $\mathrm{CR}=$ Críticamente amenazada; $\mathrm{EN}=$ en peligro; $\mathrm{V}=$ vulnerable; $\mathrm{LC}: \mathrm{NT}=$ en menor riesgo). CITES: apéndice según CITES ( $\mathrm{I}$ = podrían ser extinguidas por el tráfico; $\mathrm{II}$ = podrían extinguirse si no se controla el tráfico; III = reguladas por algún socio del tratado).

Taxa Fuente

\section{DIST}

NOM UICN-CITES

Orden Didelphimorphia

Familia Marmosidae

Marmosa canescens

1

MX

Familia Caluromyidae

Caluromys derbianus

1

NT

$\operatorname{Pr}$

Familia Didelphidae

Didelphis marsupialis

Didelphis virginiana

$1 \quad$ NT

Philander opossum

3,5

1,3

NS

Orden Xenarthra

Familia Dasypodidae

Dasypus novemcinctus

1

NS

Familia Myrmecophagidae

Tamandua mexicana

1,3

NT

A 
APÉNDICE Cont...

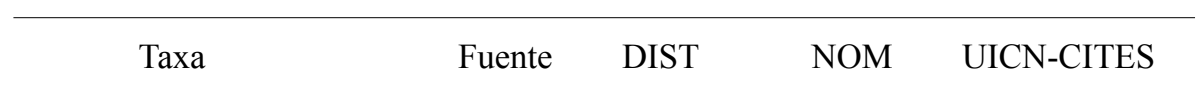

Orden Insectivora

Familia Soricidae

Criyptotis goldmani

$1 \quad$ MA

Orden Chiroptera

Familia Emballonuridae

Balantiopteryx plicata

$5 \quad$ NS

Saccopteryx bilineata

2,3 NT

Saccopteryx leptura

2 NT

Familia Noctilionidae

Noctilio leporinus

$3 \quad$ NT

Familia Mormoopidae

Mormoops megalophylla

Pteronotus davyi

Pteronotus parnellii

$\begin{array}{ll}3 & \text { NS } \\ 1,5 & \text { NT } \\ 1 & \text { NT }\end{array}$

Familia Phyllostomidae

Anoura geoffroyi
Echistenes hartii
Artibeus jamaicensis
Artibeus lituratus
Dermanura phaeotis
Carollia brevicauda
Carollia perspicillata
Carollia subrufa
Chiroderma salvini
Chiroderma villosum
Choeroniscus godmani
Chrotopterus auritus
Desmodus rotundus

2 NT

2 NT

$\operatorname{Pr}$

$1,3,4,5 \quad$ NT

$1,4 \quad$ NT

3 NT

$1,3 \quad$ NT

$1,3 \quad$ NT

$1,4,5,7$ MA

1 NT

1,2,3 NT

2 NT

$\begin{array}{ll}1 & \text { NT } \\ 1,3,6 & \text { NT }\end{array}$

MA 
APÉNDICE Cont...

\begin{tabular}{|c|c|c|c|c|}
\hline Taxa & Fuente & DIST & NOM & UICN-CITES \\
\hline Diphylla ecaudata & 1 & NS & & \\
\hline Glossophaga commissarisi & 1,2 & NT & & \\
\hline Glossophaga leachii & 5,6 & MA & & \\
\hline Glossophaga morenoi & 1,2 & MX & & \\
\hline Glossophaga soricina & $1,3,6,7$ & NT & & \\
\hline Hylonycteris underwoodi & 1 & MA & & \\
\hline Micronycteris megalotis & 5,7 & NT & & \\
\hline Micronycteris sylvestris & 1 & NT & & \\
\hline Phyllostomus discolor & 2,3 & NT & & \\
\hline Sturnira lilium & 1 & NT & & \\
\hline Sturnira ludovici & 1,3 & NT & & \\
\hline Uroderma bilobatum & $2,3,8$ & NT & & \\
\hline Uroderma magnirostrum & 2 & NT & & \\
\hline Vampyressa pusilla & 1 & NT & & \\
\hline \multicolumn{5}{|l|}{ Familia Vespertilionidae } \\
\hline Eptesicus brasiliensis & 3 & NT & & VU \\
\hline Eptesicus furinalis & 3 & NT & & \\
\hline Lasiurus borealis & 3 & EA & & \\
\hline Lasiurus ega & 3 & EA & & \\
\hline Lasiurus intermedius & 3 & NS & & \\
\hline Myotis elegans & 2,3 & MA & & \\
\hline Myotis keaysi & 2 & NT & & \\
\hline Rhogeessa tumida & 2,5 & NT & & \\
\hline \multicolumn{5}{|l|}{ Familia Molossidae } \\
\hline Molossus ater & $1,3,5$ & NT & & \\
\hline Nyctinomops laticaudatus & 2 & NT & & \\
\hline \multicolumn{5}{|l|}{ Familia Natalidae } \\
\hline Natalus stramineus & 3 & NS & & \\
\hline \multicolumn{5}{|l|}{ Orden Primates } \\
\hline Familia Cebidae & & & & \\
\hline
\end{tabular}


APÉNDICE Cont...

$\begin{array}{llll}\text { Taxa } & \text { Fuente DIST NOM UICN-CITES }\end{array}$

Ateles geoffroyi

1

MA

P VU, II

Orden Lagomorpha

Familia Leporidae

Sylvilagus floridanus

1,4 NS

Orden Rodentia

Familia Sciuridae

Sciurus aureogaster

Sciurus deppei

1,4 NT

Glaucomys volans

1 MA

1 NS

A

Familia Geomyidae

Orthogeomys grandis

3 MA

Familia Heteromyidae

Heteromys desmarestianus

$\begin{array}{lc}1 & \text { NT } \\ 1,4,5,6 & \text { MA } \\ 3,5 & \text { MA }\end{array}$

Liomys pictus

Liomys salvini

Familia Muridae

Baiomys musculus

Neotoma mexicana

1,4,5,6 MA

Olygoryzomys fulvescens

$1 \quad \mathrm{NS}$

4,6 NT

Oryzomys palustris

$4 \quad \mathrm{NS}$

Peromyscus boylii

$5 \quad \mathrm{NS}$

Peromyscus melanophrys

1,4,5 MX

Peromyscus mexicanus

Reithrodontomys fulvescens

$\begin{array}{ll}1 & \text { MA } \\ 4 & \text { EA }\end{array}$


APÉNDICE Cont...

\begin{tabular}{llll}
\hline Taxa & Fuente & DIST & NOM UICN-CITES
\end{tabular}

Reithrodontomys mexicanus

Sigmodon hispidus

Sigmodon mascotensis

\begin{tabular}{ll}
\multicolumn{1}{c}{4} & NT \\
3,4 & NS \\
3 & MX
\end{tabular}

Familia Erethizonthidae

Sphiggurus mexicanus

1 MA

A

III

Familia Agoutidae

Agouti paca

$1 \quad$ NT

III

Familia Dasyproctidae

Dasyprocta mexicana

Dasyprocta punctata

$1 \quad$ NT

III

Orden Carnivora

Familia Canidae

Urocyon cinereoargenteus

1,4,5 NS

Canis latrans

$1 \quad$ EA

Familia Procyonidae

Bassariscus sumichrasti

Nasua narica

1 MA

$1,8 \quad$ NS

Potos flavus

$1 \quad$ NT

Procyon lotor

$1 \quad$ NS

Familia Mustelidae

Conepatus mesoleucus

$1 \quad$ NS

NS I

Eira barbara

$1 \quad$ NT

P

EN, III

Mephytis macroura

NS 
APÉNDICE Cont...

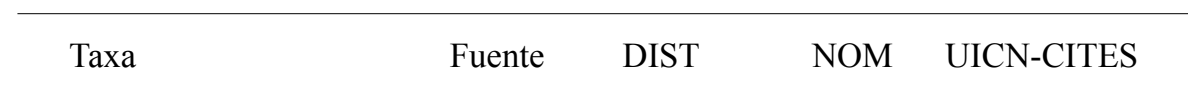

Spilogale putorius

Mustela frenata

Lontra longicaudis

Familia Felidae

Herpailurus yagouaroundi

Leopardus pardalis

Leopardus wiedii

Panthera onca

Puma concolor

Orden Perissodactyla

Familia Tapiridae

Tapirus bairdii

Orden Artiodactyla

Familia Tayassuidae

Pecari tajacu

Familia Cervidae

Mazama americana

Odocoileus virginianus
$1 \quad$ NS

$2,5 \quad \mathrm{NT}$

NT

A

II

A EN, I

P EN, I

P VU, I

P VU I

NS

NS

NS

NS

1

$\begin{array}{llll}1 & \text { NT } & \text { P } & \text { VU, I }\end{array}$

$\begin{array}{llll}1 & \text { NT } & \text { P } & \text { VU, I }\end{array}$

$\begin{array}{llll}1 & \text { NT } & \text { P } & \text { VU, I }\end{array}$

$1 \quad$ NS

II 


\begin{tabular}{|c|c|c|}
\hline $\mathrm{N}^{\circ}$ & Acrónimo de Colecciones & Nombre \\
\hline 1 & IHNMASTO & $\begin{array}{l}\text { Colección Zoológica Regional del Instituto de Historia } \\
\text { Natural. Colección Mastozoológica. }\end{array}$ \\
\hline 2 & ECO.SC.M. & $\begin{array}{l}\text { Colección Mastozológica de El Colegio de la Frontera } \\
\text { Sur. San Cristóbal de las Casas. Chiapas. }\end{array}$ \\
\hline 3 & IBUNAM & $\begin{array}{l}\text { Colección Mastozológica del Instituto de Biología de } \\
\text { la Universidad Nacional Autónoma de México. }\end{array}$ \\
\hline 4 & & Base de datos del Atlas Mastozoológico de México. \\
\hline 5 & ENCB & $\begin{array}{l}\text { Escuela Nacional de Ciencias Biológicas, Instituto } \\
\text { Politécnico Nacional. }\end{array}$ \\
\hline 6 & IUMNH & Illinois University, Museum of Natural History. \\
\hline 7 & UMMZ & $\begin{array}{l}\text { University of Michigan Museum of Zoology } \\
\text { Michigan. }\end{array}$ \\
\hline 8 & FMNH & Field Museum of Natural History, Chicago. \\
\hline 9 & KU & Kansas University, Museum of Zoology. \\
\hline 10 & MZFC & $\begin{array}{l}\text { Museo de Zoología “Alfonso L. Herrera” de la } \\
\text { Facultad de Ciencias de la Universidad Nacional } \\
\text { Autónoma de México. }\end{array}$ \\
\hline 11 & Texas A\&M & $\begin{array}{l}\text { Texas Comparative Wildlife Collection. Departament } \\
\text { of Wildlife Science, Texas A\&M. }\end{array}$ \\
\hline
\end{tabular}

